\title{
A NECESSARY AND SUFFICIENT CONDITION THAT A SUMMABILITY METHOD BE STRONGER THAN CONVERGENCE
}

\section{ALBERT WILANSKY}

Hill (Duke Math. J. vol. 9 (1942) p. 379), Agnew (Bull. Amer. Math. Soc. vol. 52 (1946) p. 128), and others have been concerned with finding conditions that summability methods should sum the convergent sequences and some others, or no others. We restrict ourselves to reversible matrix methods, that is, those in which the matrix $A=\left(a_{n k}\right)$ transforms the sequence $x=\left\{x_{n}\right\}$ into the sequence $A x$ $=\left\{A_{n}(x)\right\}=\left\{\sum_{k=0}^{\infty} a_{n k} x_{k}\right\}$ and if $y$ is any given convergent sequence, the equation $y=A x$ has a unique solution $x$. Our results are particularly simple in the special case of normal matrices, that is, those with $a_{n k}=0$ for $k>n, a_{n n} \neq 0$.

We deal with conservative matrices, that is, those which transform every convergent sequence into a convergent sequence. The regular matrices form a subclass.

Consider, for the moment, normal matrices. What is known, and indeed trivial, is the following: a normal conservative matrix $A$ sums only convergent sequences if and only if $A^{-1}$ is conservative. Now, setting $\|A\|=\sup _{n} \sum_{k=0}^{\infty}\left|a_{n k}\right|, A$ is conservative if and only if $\|A\|<\infty$ and in addition the sequences formed from its columns, and the sequence $\left\{\sum_{k=0}^{\infty} a_{n k}\right\}$, are all convergent (Silverman-ToeplitzKojima-Carmichael et al.). Thus the necessary and sufficient conditions are transparently displayed. What we propose to do is reduce these conditions by two, keeping the one which the author has found easiest to check in certain special cases.

According to Banach, Theorie des operations lineaires, Warsaw, 1932, p. 50, if $A$ is reversible there is a bounded sequence $\left\{c_{k}\right\}$ and a matrix $B=\left(b_{n k}\right)$ such that if $y$ is convergent then $y=A x$ where $x=\left\{x_{n}\right\}, x_{n}=c_{n} \lim _{r} y_{r}+\sum_{k=0}^{\infty} b_{n k} y_{k}$, the series being always convergent. Clearly if $A$ is normal, $c_{n}=0$ and $B=A^{-1}$. As one of several possibilities let us define $\left\|A^{-1}\right\|$ as $\sup _{n}\left\{\left|c_{n}\right|+\sum_{k=0}^{\infty}\left|b_{n k}\right|\right\}$. This is consistent with the case when $A$ is normal.

Theorem. $A$ reversible conservative matrix $A$ sums a divergent sequence if and only if $\left\|A^{-1}\right\|=\infty$.

In particular if $\left\|A^{-1}\right\|<\infty$ we automatically conclude the convergence of the above mentioned sequences (formed from $A^{-1}$ ).

Received by the editors May 20, 1948, and, in revised form, July 28, 1948. 
Sufficiency. Suppose $\left\|A^{-1}\right\|=\infty$. Since $\left\{c_{n}\right\}$ is bounded this means that $\|B\|=\infty$. By a now classical scheme we may construct a convergent sequence $y$ such that $B y$ is unbounded. Let $x_{n}=c_{n} \lim _{r} y_{r}$ $+\sum_{k=0}^{\infty} b_{n k} y_{k}$. The sequence $x$ is also unbounded since $\left\{c_{n}\right\}$ is bounded but $A x=y$ is convergent.

Necessity. Suppose $\left\|A^{-1}\right\|<\infty$. Let $x$ be summable $A$. Then $y=A x$ is convergent and $\left|x_{n}\right|=\left|c_{n} \lim y_{r}+\sum b_{n k} y_{k}\right| \leqq|| A^{-1}|| \cdot \sup \left|y_{r}\right|<\infty$ by hypothesis and since $y$ is convergent. Thus $x$ is a bounded sequence. The result is then immediate from the following lemma.

Lemma. If a reversible conservative matrix sums only bounded sequences it sums only convergent sequences.

A proof is given for perfect matrices by Hill (Bull. Amer. Math. Soc. vol. 50 (1944) p. 229). The following proof for conservative matrices while still leaning on the methods of Mazur and Banach is along different lines.

Suppose first that $A$ is an arbitrary reversible matrix. We denote its convergence field by (A). For a sequence $x$ define $\|x\|=\sup _{n}\left|x_{n}\right|$. We shall call this the first metric. The second metric will be that defined by $\|x\|=\sup _{n}\left|A_{n}(x)\right|$. The one to one correspondence $x \leftrightarrow A x$ between $(A)$ and $(c)$ (the class of convergent sequences) respectively is norm-preserving using the second and first metrics respectively and so $(A)$ is a separable Banach space using the second metric. Moreover suppose $\left\{x^{r}\right\}$ is a sequence of elements of $(A)$ with limit $x$ in $(A)$ using the second metric. We prove that

$$
\lim _{r \rightarrow \infty} x_{k}^{r}=x_{k} \quad \text { for each } k .
$$

For a fixed $t$, define $F(y)=y_{t}$ where $y$ is any sequence. Let $D=\left(d_{n k}\right)$ where $d_{n k}=0$ for $k \neq t, d_{n t}=1$. Then the matrix $D$ sums any sequence $y$ whatsoever to the limit $y_{t}=F(y)$. Since $(D) \supseteq(A)$ the functional $F$ is linear and continuous on $(A)$ by use of the second metric by a result of Mazur. (Studia Math. vol. 2 (1930) p. 42. For this result Mazur uses only the hypothesis that $(A)$ is a Banach space.) This proves (1).

The above results have been derived using only the reversibility of A.

Imposing the hypotheses of the lemma on $A$ we next show that $(A)$ is a Banach space using the first metric. We have only to show that it is complete so let us suppose that a sequence $\left\{x^{r}\right\}$ of elements of $(A)$ is a Cauchy sequence using the first metric. It is readily verified that it is then a Cauchy sequence using the second metric. Thus $\left\{x^{r}\right\}$ converges to an element $x$ of $(A)$ using the second metric. It 
follows from (1) that the bounded sequence to which $\left\{x^{r}\right\}$ converges using the first metric is actually $x$. Thus $(A)$ is a Banach space using either metric. It follows (Banach, loc. cit. Theorem 6, p. 41) that the two metrices are equivalent. But, as mentioned above, $(A)$ is separable if we use the second metric and so it is separable if we use the first metric, and our lemma follows from a result of Agnew (Ann. of Math. (2) vol. 46 (1945) line 8 from foot of p. 99) that the bounded field of a matrix is not separable using the first metric unless the matrix sums no bounded divergent sequences.

LEHIGH UNIVERSITY 